TRANI, P.E.; PASSOS, F.A.; FOLTRAN, D.E.; TIVELLI, S.W.; RIBEIRO, I.J.A. Avaliação dos acessos de alho pertencentes à coleção do Instituto Agronômico de Campinas. Horticultura Brasileira, Brasília, v.23, n.4, p.935-939, out-dez 2005.

\title{
Avaliação dos acessos de alho coleção do Instituto Agronômico de Campinas
}

\author{
Paulo E. Trani ${ }^{1}$; Francisco A. Passos ${ }^{1}$; Dulcinéia E. Foltran² ${ }^{2}$ Sebastião W. Tivelli ${ }^{1}$; Ivan J.A. Ribeiro ${ }^{3}$ \\ ${ }^{1}$ Instituto Agronômico, Centro de Horticultura, C. Postal 28, 13012-970 Campinas-SP; E-mail: petrani@iac.sp.gov.br; ${ }^{2}$ APTA-UPD de \\ Tietê, C. Postal 18, 18530-970 Tietê-SP; E-mail: dulcineia@aptaregional.sp.gov.br; ${ }^{3}$ Instituto Agronômico, C. Postal 28, $13012-970$ \\ Campinas-SP
}

\section{RESUMO}

Cinqüenta acessos de alho pertencentes ao banco de germoplasma do Instituto Agronômico de Campinas foram avaliados em campo, nos municípios de Tietê e Jundiaí (SP), Brasil, em 2003, em blocos ao acaso, com três repetições. Os dados obtidos foram submetidos à análise de variância, sendo as médias comparadas pelo teste de Skott-Knott, em nível de 5\% de probabilidade. Os acessos Piedade e Gigante de Curitibanos apresentaram excelente desempenho para produtividade e qualidade do bulbo, nos dois locais. Por outro lado, os acessos Bulbilho Aéreo 2 e Andradas Manoel Lopes 2 apresentaram interação com o ambiente, o primeiro para o tamanho do bulbo e o segundo para a produtividade. Comparando-se os resultados de Jundiaí com os obtidos em Tietê, nota-se que em Jundiaí houve maior variabilidade genética para a produtividade e para o diâmetro do bulbo, devido ao menor efeito ambiental.

Palavras-chave: Allium sativum L., banco de germoplasma, produtividade.

\begin{abstract}
Evaluation of garlic accesses from the collection of the Instituto Agronômico de Campinas

Fifty accesses from the garlic germplasm bank of Instituto Agronômico de Campinas were evaluated in Tietê and Jundiaí, São Paulo State, Brazil. The field trials were carried out in 2003, and the experimental design was randomized blocks with three replicates. The data were subjected to an ANOVA and the means compared using the Skott-Knott test at a 5\% probability level. The access Piedade and Gigante de Curitibanos showed excellent performance for yield and bulb quality in both locations. On the other hand, environmental interaction occurred for access Bulbilho Aéreo 2 and Andradas Manoel Lopes 2. For the first one, the bulb size was different and for the second the yield changed. A greater genetic diversity on yield and bulb diameter was observed in Jundiaí, due to lower environmental effects.
\end{abstract}

Keywords: Allium sativum L., germplasm bank, yield.

(Recebido para publicação em 4 de novembro de 2004 e aceito em 3 de agosto de 2005)

$\mathrm{O}$ Brasil produziu $114.436 \mathrm{t}$ de alho em 15.715 ha em 2002 (IBGE, 2002), para um consumo estimado em 140 mil toneladas neste mesmo ano (DELLAMARIA, 2003). Segundo Camargo Filho (2003) a produtividade de alho no estado de São Paulo foi de aproximadamente $6,4 \mathrm{t} \mathrm{ha}^{-1} \mathrm{em} 2003$. Os acessos do banco de germoplasma do Instituto Agronômico de Campinas (IAC) têm revelado produtividades superiores a esta média.

O Centro de Horticultura do IAC mantém um banco de germoplasma de alho com 50 acessos e realiza experimentos regionais com esse germoplasma desde a década de 40 . Dentre os acessos mantidos nessa coleção, encontram-se germoplasmas seminobres que podem ser utilizados por produtores em substituição aos alhos nobres. Os alhos semi-nobres são recomendados para o plantio em São Paulo pois são mais adaptados às condições de fotoperíodo e temperatura, dispensam o tratamento de vernalização, são produtivos e de boa qualidade comercial. Os bulbos e bulbilhos desses acessos são arroxeados, porém, são mais claros do que os do alho nobre. O número de bulbilhos por bulbo é de 15 em média e não produzem palitos (CAMARGO FILHO et al., 1996; SIQUEIRA et al., 1996).

São indicados para plantio em São Paulo as cvs. Amarante, Chinês, Gigante de Curitibanos, Lavínia e Roxinho (TAVARES et al., 1998).

A expansão da área cultivada com alhos nobres e roxos ocorrida na década de 80 e 90 no Brasil em áreas novas ou que tradicionalmente cultivavam os alhos comuns e brancos, aumentou o custo de produção da cultura especialmente para a aquisição de semente. $\mathrm{O}$ custo de produção por hectare, de alho vernalizado, para São Paulo, foi de US\$6.070,00 (março/1995: $\mathrm{R} \$ 1,00=$ US\$0,90) (TRANI et al., 1997). Este alto custo foi semelhante ao valor observado em Santa Catarina, de US $\$ 6.946,00$ (CARMARGO FILHO et al., 1996). Nos coeficientes técnicos para a cultura do alho vernalizado, o custo da semente representou $51 \%$ do valor dos insumos ou $33 \%$ do custo total, enquanto o custo de vernalização foi de cerca de $16 \%$ do preço da semente ou 5\% do custo total (TRANI et al., 1997). Esse custo elevado dificulta o cultivo de alhos nobres para os pequenos produtores e produtores familiares de diversas regiões brasileiras.

A cultura do alho é uma atividade econômica importante no sistema de agricultura familiar. Além de empregar mão-de-obra desde o plantio até a colheita, permite a utilização da mão-deobra num período que essa estaria ociosa. Com isto, favorece a fixação do homem ao campo e proporciona uma renda extra para a família.

As mutações somáticas e a seleção cuidadosa dos produtores deram origem 
a um grande número de cultivares de alhos semi nobres e comuns com características agronômicas distintas. Estas cultivares são exploradas economicamente no sistema de agricultura familiar nas distintas regiões produtoras de São Paulo, as quais apresentam características edafoclimáticas diferentes.

Em 1987, Lisbão et al. (1993) avaliaram o comportamento de acessos de alho em três municípios paulistas (Monte Alegre do Sul, Pariquera-Açu e Guatapará). Os acessos estudados apresentaram comportamento diferente em cada município. Em Monte Alegre do Sul, os acessos IAC Lavínia 1632 (9,2 t $\left.\mathrm{ha}^{-1}\right)$, Roxinho I-5063 (6,3 $\left.\mathrm{t} \mathrm{ha}^{-1}\right)$ e Peruano $\left(5,6 \mathrm{t} \mathrm{ha}^{-1}\right)$ foram superiores aos demais, enquanto em Pariquera-Açu os mais produtivos foram Santa Catarina Roxo (4,9 t ha $\left.{ }^{-1}\right)$; Cajuru I-2315 (4,9 t ha $\left.{ }^{-1}\right)$; São José $4999\left(4,5 \mathrm{t} \mathrm{ha}^{-1}\right)$ e Areal no 2 I-3978 (4,4 t ha-1). Nenhum dos acessos avaliados em Pariquera-Açu obteve produtividade superior a 5,0 t ha-1 devido às condições climáticas locais. Por outro lado, os acessos mais produtivos em Guatapará foram o Peruano $\left(7,2 \mathrm{t} \mathrm{ha}^{-1}\right)$ e o Chinês $\left(4,8 \mathrm{t} \mathrm{ha}^{-1}\right)$.

No município paulista de Tietê, Lisbão et al. (1991) avaliaram o comportamento de dez acessos de alho, em 1988. O acesso Lavínia foi o mais produtivo entre os dez testados, com produtividade de $12,8 \mathrm{t} \mathrm{ha}^{-1}$. Os demais acessos revelaram produtividades superiores à cultivar tradicionalmente cultivada na região (Roxinho de Goiânia), que produziu apenas 1,7 $\mathrm{t} \mathrm{ha}^{-1}$. Dentre os outros acessos merecem ser destacados os genótipos Chinês, Mineiro e Roxinho, com produtividades de 8,1; 7,9 e 7,7 t ha-1, respectivamente.

Como parte do programa de pesquisa e adaptação de tecnologias regionais, o IAC, em conjunto com a CATI, estudaram o comportamento varietal do alho em Guatapará em 1988 e em Ribeirão Preto no ano seguinte. Os resultados de 1989 em Ribeirão Preto confirmaram os dados do ano anterior obtidos em Guatapará. As médias de produtividade e peso médio dos bulbos nos dois anos de estudo, demostraram que os acessos Lavínia (12,3 t ha-1 e 28,8 g), Cateto Roxo (10,7 t ha-1 e 27,5 g), Roxinho $\left(10,7 \mathrm{t} \mathrm{ha}^{-1}\right.$ e $\left.25,0 \mathrm{~g}\right)$ e Chinês $\left(9,1 \mathrm{t} \mathrm{ha}^{-1}\right.$ e 24,1 g) foram superiores aos demais acessos avaliados (LISBÃO et al., 1991; LISBÃO et al., 1993).

Segundo Siqueira et al. (1996), o acesso Assaí (IAC-3702) foi identificado no banco de germoplasma do IAC, como sendo muito semelhante ao acesso Cateto Roxo. Portanto, ele pode substituir o acesso Cateto Roxo com vantagens, pois seus bulbos apresentam em média 10 dentes ou bulbilhos, enquanto no Cateto Roxo ocorrem mais de 30 dentes. Além disso, as plantas do acesso Assaí são tolerantes ao pseudoperfilhamento. Diante disto, os pequenos produtores que cultivam Cateto Roxo para consumo próprio e vendas locais, podem passar a utilizá-lo em plantios comerciais ou experimentais.

Os resultados obtidos indicam que as produtividades dos acessos de alho são muito variáveis de região para região, de acordo com as condições edafoclimáticas e os tratos culturais empregados. O objetivo deste trabalho foi avaliar o número de bulbos por hectare, a produtividade, o peso médio e o diâmetro dos bulbos, que são relacionados à qualidade comercial de genótipos do banco de germoplasma de alho do Centro de Horticultura do IAC.

\section{MATERIAL E MÉTODOS}

Dois experimentos de campo foram instalados em duas localidades do estado de São Paulo, em 2003, para avaliação de 50 acessos de alho, pertencentes ao banco de germoplasma do Centro de Horticultura do Instituto Agronômico de Campinas.

O primeiro experimento foi instalado em 03/04/2003 em Tietê, enquanto o segundo ocorreu em 10/04/2003 em Jundiaí. Os bulbilhos de cada acesso utilizado no plantio foram selecionados em peneira $\mathrm{n}^{\circ} 2$ (peso 1,5-2,0 g). O alho foi plantado em linhas simples transversais aos canteiros, os quais tinham um metro de largura. $\mathrm{O}$ espaçamento entre as linhas foi de $0,25 \mathrm{~m}$ e, entre plantas, de $0,1 \mathrm{~m}$. Cada parcela conteve $0,5 \mathrm{~m}^{2}$ de área total e foi composta de duas linhas transversais. No experimento de Tietê cada linha foi plantada com sete bulbilhos, num total de 14 bulbilhos por parcela, ou $0,35 \mathrm{~m}^{2}$ de área cultivada.
Em Jundiaí por sua vez, cada linha de plantio nos canteiros conteve 6 bulbilhos, num total de 12 bulbilhos por parcela, ou $0,30 \mathrm{~m}^{2}$ de área cultivada. Em ambos os locais os tratos culturais (calagem, adubação, irrigação, tratos fitossanitários, e etc.) foram realizados de acordo com Trani et al. (1997).

Independentemente do ciclo, todos os acessos foram colhidos no mesmo dia em cada local. Em Tietê a colheita ocorreu em 08/09/2003, e no dia seguinte, 09/09/2003, foi colhido o experimento em Jundiaí. Em ambas as localidades, foram avaliados quatro caracteres: número de bulbos por parcela, produtividade $\left(\mathrm{t} \mathrm{ha}^{-1}\right)$, peso médio do bulbo $(\mathrm{g})$ e diâmetro médio do bulbo ( $\mathrm{mm})$. $\mathrm{O}$ diâmetro médio do bulbo foi obtido por meio da média aritmética de três bulbos por parcela escolhidos ao acaso.

Para efeito de comparação dos acessos quanto à produtividade em 2003 com os resultados obtidos em experimentos anteriores, os dados de peso total de bulbos em g parcela ${ }^{-1}$ no experimento de Tietê/SP foram convertidos para $\mathrm{t} \mathrm{ha}^{-1}$ por meio da equação Produtividade $_{\text {Tietê }}\left(\mathrm{t} \mathrm{ha}^{-1}\right)=(2,857 *$ peso total de bulbos (g parcela $\left.{ }^{-1}\right) *$ 0,007. Por motivos idênticos, os dados de peso total de bulbos em $g$ parcela ${ }^{-1}$ no experimento de Jundiaí/SP foram convertidos para t ha ${ }^{-1}$ por meio da equação Produtividade Jundiaí $\left(\mathrm{t} \mathrm{ha}^{-1}\right)=(3,333$ * peso total de bulbos (g parcela $\left.{ }^{-1}\right) *$ 0,007. Considerou-se que um hectare de cultivo de alho corresponde a $7.000 \mathrm{~m}^{2}$ de canteiros, o que origina o fator 0,007.

Os experimentos foram conduzidos em blocos ao acaso com três repetições. Os dados obtidos foram submetidos ao teste de Hartley, em nível de 5\% de probabilidade, para verificar a homogeneidade de variâncias. Em ambos os experimentos os dados originais foram submetidos à análise de variância, e tiveram as médias comparadas pelo teste de Scott-Knott, em nível de 5\% de probabilidade (FERREIRA, 2005).

\section{RESULTADOS E DISCUSSÃO}

Devido à heterogeneidade dos quadrados médios residuais (GOMES, 1970), os resultados dos experimentos são apresentados separadamente. 
Para a variável número de bulbos por hectare não houve efeito significativo de acessos no experimento de Tietê em razão da uniformidade de emergência dos bulbilhos, ocorrendo reduzido número de falhas (Tabela 1). Tanto na análise de variância quanto no teste de ScottKnott não houve diferenças significativas em Tietê.

Por outro lado, na análise de variância, para o experimento em Tietê observou-se que houve efeito altamente significativo de acessos para os caracteres produtividade, peso médio do bulbo e diâmetro médio do bulbo. O peso médio do bulbo, que está relacionado ao tamanho ou volume do mesmo, foi o principal fator a influenciar a produtividade. Os coeficientes de variação para a produtividade, peso médio do bulbo e diâmetro médio do bulbo, foram $18,4 \%$, $16,6 \%$ e $8,0 \%$, respectivamente (Tabela 1).

Em Tietê foram detectadas cinco classes para a produtividade. $\mathrm{O}$ acesso Bulbilho Aéreo 2 com produtividade de 14,9 $\mathrm{t} \mathrm{ha}^{-1}$, e o acesso Piedade com produtividade de 13,4 $\mathrm{t} \mathrm{ha}^{-1}$, superaram estatisticamente os demais. Na segunda classe ficaram os acessos Gigante de Curitibanos e Andradas Manoel Lopes 2 , que produziram $10,9 \mathrm{tha}^{-1} \mathrm{e} 10,1 \mathrm{t} \mathrm{ha}^{-1}$, respectivamente. $\mathrm{O}$ acesso Gigante de Curitibanos foi o padrão de referência neste experimento devido a sua expressão no cultivo comercial.

Comparando a produtividade de acessos do experimento em Tietê com os obtidos por Lisbão et al. (1991) no mesmo local, duas considerações merecem ser destacadas. Primeiro, a maior produtividade obtida no ano de 1988 pelos autores com o acesso Lavínia foi de $12,8 \mathrm{tha}^{-1}$, o que não é muito diferente das produtividades expressas no presente experimento para os acessos Bulbilho Aéreo 2 e Piedade. Segundo, os acessos de melhor produtividade no trabalho de Lisbão et al. (1991) revelaram uma produtividade bem abaixo no experimento atual, com exceção de Mineiro que havia produzido $7,9 \mathrm{t} \mathrm{ha}^{-1} \mathrm{e}$ agora produziu 7,6 $\mathrm{t} \mathrm{ha}^{-1}$. Este resultado pode ser explicado pela interação entre genótipo e ambiente.

Resultados semelhantes às produtividades dos acessos foram obtidos para
Tabela 1. Médias do número de bulbos ( $\mathrm{mil} \mathrm{ha}^{-1}$ ), produtividade ( $\mathrm{t}$ ha), peso médio do bulbo (g), e diâmetro médio do bulbo ( $\mathrm{mm}$ ) de 50 acessos de alho do banco ativo de Germoplasma do IAC em Tietê, IAC, 2003.

\begin{tabular}{|c|c|c|c|c|}
\hline Acesso & $\begin{array}{l}\text { Número de } \\
\text { bulbos } \\
\left(\mathrm{mil} \mathrm{ha}^{-1}\right)^{1}\end{array}$ & $\begin{array}{l}\text { Produtividade } \\
\text { (t ha })^{1}\end{array}$ & $\begin{array}{l}\text { Peso médio } \\
\text { do bulbo }(g)^{1}\end{array}$ & $\begin{array}{c}\text { Diâmetro } \\
\text { médio do } \\
\text { bulbo }(\mathrm{mm})^{1,2}\end{array}$ \\
\hline Bulbilho Aéreo 2 & 266,0 a & $14,9 a$ & 55,8 a & $48,9 \mathrm{a}$ \\
\hline Piedade & $280,0 \mathrm{a}$ & $13,4 \mathrm{a}$ & $47,9 \mathrm{~b}$ & 49,3 a \\
\hline Gigante de Curitibanos & 266,0 a & $10,9 \mathrm{~b}$ & $40,8 \mathrm{c}$ & $44,6 \mathrm{~b}$ \\
\hline Andradas Manoel Lopes 2 & 274,0 a & $10,1 \mathrm{~b}$ & $36,9 \mathrm{c}$ & $42,3 \mathrm{~b}$ \\
\hline BGH 5952 & 300,0 a & $8,6 \mathrm{c}$ & $28,9 d$ & $39,8 \mathrm{~b}$ \\
\hline Mendonça 5062 & 280,0 a & $8,4 \mathrm{c}$ & $30,1 \mathrm{~d}$ & $37,9 \mathrm{c}$ \\
\hline Roxo Capim & 280,0 a & $8,2 \mathrm{c}$ & $29,1 \mathrm{~d}$ & $37,5 \mathrm{c}$ \\
\hline Catetinho do Paraná & 274,0 a & $7,9 \mathrm{c}$ & $28,8 d$ & $36,1 \mathrm{c}$ \\
\hline Mineiro & 280,0 a & $7,6 \mathrm{c}$ & $27,3 d$ & $36,2 \mathrm{c}$ \\
\hline Tatuí 3705 & 266,0 a & $7,6 \mathrm{c}$ & $28,3 d$ & $37,5 \mathrm{c}$ \\
\hline Canela de Ema & 280,0 a & $7,2 \mathrm{~d}$ & $25,7 d$ & $35,4 \mathrm{c}$ \\
\hline BGH 5935 & 280,0 a & $6,8 \mathrm{~d}$ & $23,7 \mathrm{e}$ & $34,0 \mathrm{~d}$ \\
\hline BGH 5947 & 294,0 a & $6,4 \mathrm{~d}$ & 21,7 e & $35,0 \mathrm{c}$ \\
\hline Bom Repouso & 266,0 a & $6,4 \mathrm{~d}$ & 23,8 e & $36,5 \mathrm{c}$ \\
\hline Santa Catarina Roxo & 266,0 a & $6,3 d$ & 23,5 e & $36,4 \mathrm{c}$ \\
\hline Gigante Tietê & 280,0 a & $6,2 \mathrm{~d}$ & 22,3 e & $35,2 \mathrm{c}$ \\
\hline Cateto Precoce I-99 & 274,0 a & $6,2 \mathrm{~d}$ & 22,7 e & $36,2 \mathrm{c}$ \\
\hline Cateto Roxo & 294,0 a & $6,0 \mathrm{~d}$ & 20,3 e & $32,3 d$ \\
\hline São José 4999 & 274,0 a & $5,8 d$ & 21,3 e & $33,8 \mathrm{~d}$ \\
\hline BGH 5963 & 266,0 a & $5,8 d$ & 21,9 e & $33,3 d$ \\
\hline Peruano Bisão & $280,0 \mathrm{a}$ & $5,7 d$ & 20,3 e & $32,4 \mathrm{~d}$ \\
\hline BGH 4842 & 266,0 a & $5,6 d$ & 21,3 e & $32,5 \mathrm{~d}$ \\
\hline Santa Catarina Branco & $280,0 \mathrm{a}$ & $5,6 d$ & 20,2 e & $33,3 d$ \\
\hline Lavínia 3208 & 280,0 a & $5,4 d$ & $19,3 \mathrm{f}$ & $32,7 \mathrm{~d}$ \\
\hline BGH 5936 & $280,0 \mathrm{a}$ & $5,3 d$ & $19,2 f$ & $32,2 \mathrm{~d}$ \\
\hline Areal n² 3978 & 260,0 a & $5,3 d$ & 20,5 e & $31,5 \mathrm{e}$ \\
\hline BGH 6394 & 246,0 a & $5,1 d$ & $20,8 \mathrm{e}$ & $33,3 \mathrm{~d}$ \\
\hline Cará & 266,0 a & 5,0 e & $18,8 \mathrm{f}$ & $33,1 \mathrm{~d}$ \\
\hline Sacaia Goiânia & 294,0 a & $5,0 \mathrm{e}$ & $16,9 f$ & $33,0 \mathrm{~d}$ \\
\hline BGH 4823 & 266,0 a & $4,8 \mathrm{e}$ & $18,1 \mathrm{f}$ & $32,2 \mathrm{~d}$ \\
\hline Chinês Esalq & 286,0 a & $4,7 \mathrm{e}$ & $16,5 f$ & 30,4 e \\
\hline Vera Cruz 5004 & $280,0 \mathrm{a}$ & $4,7 \mathrm{e}$ & $16,9 \mathrm{f}$ & $33,0 \mathrm{~d}$ \\
\hline Do Reino de Araras & 274,0 a & 4,7 e & $17,3 f$ & 31,0 e \\
\hline Formosa 4713 & 274,0 a & 4,7 e & $17,3 \mathrm{f}$ & 31,0 e \\
\hline Andradas Manoel Lopes 1 & 274,0 a & $4,6 \mathrm{e}$ & $16,8 f$ & $32,7 d$ \\
\hline Roxo Araras & 274,0 a & $4,6 \mathrm{e}$ & $16,8 \mathrm{f}$ & 30,6 e \\
\hline Chinês 4653 & 286,0 a & $4,6 \mathrm{e}$ & $16,0 \mathrm{f}$ & 30,2 e \\
\hline Mexicano & 266,0 a & $4,5 \mathrm{e}$ & $16,8 \mathrm{f}$ & $32,3 \mathrm{~d}$ \\
\hline Lavínia 1632 & 274,0 a & $4,2 \mathrm{e}$ & $15,6 \mathrm{f}$ & 29,2 e \\
\hline Gravataí & 266,0 a & $4,2 \mathrm{e}$ & $15,8 f$ & 31,2 e \\
\hline Assaí 3702 & 254,0 a & $4,1 \mathrm{e}$ & $16,3 f$ & 29,4 e \\
\hline BGH 0525 & 286,0 a & $4,0 \mathrm{e}$ & $14,2 f$ & $27,5 \mathrm{f}$ \\
\hline Roxinho 5063 & 280,0 a & 3,9 e & $13,8 \mathrm{f}$ & $28,3 \mathrm{f}$ \\
\hline Alho Preto & $280,0 \mathrm{a}$ & $3,9 \mathrm{e}$ & $13,8 \mathrm{f}$ & $28,5 \mathrm{f}$ \\
\hline Roxo Mineiro & 260,0 a & $3,8 \mathrm{e}$ & $14,5 \mathrm{f}$ & $25,0 \mathrm{f}$ \\
\hline Mossoró & 266,0 a & $3,8 \mathrm{e}$ & $14,1 \mathrm{f}$ & $30,8 \mathrm{e}$ \\
\hline BGH 4814 & 260,0 a & $3,5 \mathrm{e}$ & $13,6 \mathrm{f}$ & $27,8 f$ \\
\hline Amarante Embrapa & 274,0 a & $3,3 \mathrm{e}$ & $11,9 f$ & $27,0 \mathrm{f}$ \\
\hline Roxo de Araras 2 & 266,0 a & $2,9 \mathrm{e}$ & $10,6 f$ & $26,8 \mathrm{f}$ \\
\hline Cajuru 2315 & $260,0 \mathrm{a}$ & $2,5 \mathrm{e}$ & $9,8 \mathrm{f}$ & $26,3 \mathrm{f}$ \\
\hline C.V. (\%) & 7,1 & 18,4 & 16,6 & 8,0 \\
\hline $\mathrm{F}$ & $0,9 n s$ & $18,4^{* *}$ & $15,1^{* *}$ & $10,9^{* *}$ \\
\hline
\end{tabular}

${ }^{1}$ Médias seguidas pela mesma letra na coluna não diferem entre si (teste de Scott-Knott, $5 \%) ;{ }^{2}$ Baseado na média aritmética de 3 bulbos/parcela $\left(0,35 \mathrm{~m}^{2}\right)$, escolhidos ao acaso; ** Efeito de acessos $(\mathrm{P}<0,01)$; ${ }^{\text {ns }}$ Ausência de efeito de acessos. 
Tabela 2. Médias do número de bulbos ( $\mathrm{mil} \mathrm{ha}^{-1}$ ), produtividade ( $\mathrm{t}$ ha), peso médio do bulbo (g), e diâmetro médio do bulbo $(\mathrm{mm})$ de 50 acessos de alho do banco ativo de Germoplasma do IAC em Jundiaí, IAC, 2003.

\begin{tabular}{|c|c|c|c|c|}
\hline Acesso & $\begin{array}{l}\text { Número de } \\
\text { bulbos } \\
\left(\mathrm{mil} \mathrm{ha}^{-1}\right)^{1}\end{array}$ & $\begin{array}{l}\text { Produtividade } \\
\qquad(\mathrm{t} \mathrm{ha})^{1}\end{array}$ & $\begin{array}{l}\text { Peso médio } \\
\text { do bulbo }(g)^{1}\end{array}$ & $\begin{array}{c}\text { Diâmetro } \\
\text { médio do } \\
\text { bulbo }(\mathrm{mm})^{1,2}\end{array}$ \\
\hline Bulbilho Aéreo 2 & $273,0 \mathrm{a}$ & $13,1 \mathrm{a}$ & $48,0 \mathrm{a}$ & $39,3 \mathrm{c}$ \\
\hline Piedade & $287,0 \mathrm{a}$ & $11,6 \mathrm{~b}$ & $40,5 \mathrm{~b}$ & $47,3 \mathrm{a}$ \\
\hline Gigante de Curitibanos & $273,0 \mathrm{a}$ & $11,4 \mathrm{~b}$ & $41,7 \mathrm{~b}$ & $45,3 \mathrm{a}$ \\
\hline BGH 5952 & $287,0 \mathrm{a}$ & $9,8 \mathrm{c}$ & $34,3 \mathrm{c}$ & $38,3 \mathrm{c}$ \\
\hline Lavínia 1632 & $273,0 \mathrm{a}$ & $9,6 \mathrm{c}$ & $35,3 \mathrm{c}$ & $39,3 \mathrm{c}$ \\
\hline Roxo Capim & $287,0 \mathrm{a}$ & $9,3 \mathrm{c}$ & $32,4 \mathrm{c}$ & $38,7 \mathrm{c}$ \\
\hline Andradas Manoel Lopes 2 & $280,0 \mathrm{a}$ & $9,0 \mathrm{c}$ & $32,2 \mathrm{c}$ & $40,7 \mathrm{~b}$ \\
\hline BGH 5935 & $280,0 \mathrm{a}$ & $8,7 \mathrm{~d}$ & $31,0 \mathrm{~d}$ & $35,0 \mathrm{~d}$ \\
\hline Mineiro & $287,0 \mathrm{a}$ & $8,6 \mathrm{~d}$ & $29,9 d$ & $32,3 \mathrm{e}$ \\
\hline Tatuí 3705 & $280,0 \mathrm{a}$ & $8,3 d$ & $29,8 d$ & $35,3 d$ \\
\hline Gigante Tietê & $287,0 \mathrm{a}$ & $8,1 \mathrm{~d}$ & $28,3 d$ & $41,7 \mathrm{~b}$ \\
\hline BGH 4842 & $273,0 \mathrm{a}$ & $8,1 \mathrm{~d}$ & $29,8 d$ & $36,0 d$ \\
\hline Peruano Bisão & $296,3 \mathrm{a}$ & $7,5 \mathrm{e}$ & $25,3 \mathrm{e}$ & $32,7 \mathrm{e}$ \\
\hline Mendonça 5062 & $287,0 \mathrm{a}$ & $7,4 \mathrm{e}$ & 25,7 e & $37,0 \mathrm{~d}$ \\
\hline Chinês Esalq & $296,3 \mathrm{a}$ & $7,2 \mathrm{e}$ & 24,3 e & $32,3 \mathrm{e}$ \\
\hline BGH 5947 & $280,0 \mathrm{a}$ & $7,1 \mathrm{e}$ & $25,3 \mathrm{e}$ & 33,0 e \\
\hline Cateto Roxo & $326,7 \mathrm{a}$ & $7,1 \mathrm{e}$ & $21,7 \mathrm{f}$ & 30,3 e \\
\hline Areal $n^{\circ} 23978$ & $287,0 \mathrm{a}$ & $7,0 \mathrm{e}$ & $24,5 \mathrm{e}$ & $33,7 \mathrm{e}$ \\
\hline Bom Repouso & $263,7 \mathrm{a}$ & $7,0 \mathrm{e}$ & 26,7 e & $33,0 \mathrm{e}$ \\
\hline Santa Catarina Roxo & $310,3 \mathrm{a}$ & $7,0 \mathrm{e}$ & $22,6 \mathrm{f}$ & $36,3 d$ \\
\hline Lavínia 3208 & $280,0 \mathrm{a}$ & $6,9 \mathrm{e}$ & $24,6 \mathrm{e}$ & $32,0 \mathrm{e}$ \\
\hline São José 4999 & $287,0 \mathrm{a}$ & $6,7 \mathrm{f}$ & $23,3 f$ & $35,3 d$ \\
\hline Catetinho do Paraná & $287,0 \mathrm{a}$ & $6,5 \mathrm{f}$ & $22,8 \mathrm{f}$ & $33,3 \mathrm{e}$ \\
\hline Cateto Precoce I-99 & $296,3 \mathrm{a}$ & $6,5 \mathrm{f}$ & $21,9 \mathrm{f}$ & $34,7 \mathrm{~d}$ \\
\hline Mexicano & $287,0 \mathrm{a}$ & $6,5 \mathrm{f}$ & $22,5 \mathrm{f}$ & 32,7 e \\
\hline Sacaia Goiânia & $273,0 \mathrm{a}$ & $6,5 \mathrm{f}$ & $23,6 \mathrm{e}$ & $31,0 \mathrm{e}$ \\
\hline 24 & 343,0 a & $6,3 \mathrm{f}$ & $18,5 \mathrm{~g}$ & 30,3 e \\
\hline Chinês 4653 & $256,7 \mathrm{a}$ & $6,3 \mathrm{f}$ & 24,4 e & $31,7 \mathrm{e}$ \\
\hline BGH 6394 & 296,3 a & $5,9 \mathrm{f}$ & $20,1 \mathrm{~g}$ & $31,7 \mathrm{e}$ \\
\hline BGH 0525 & $263,7 \mathrm{a}$ & $5,7 \mathrm{~g}$ & $21,5 f$ & 29,7 e \\
\hline Cará & $273,0 \mathrm{a}$ & $5,6 \mathrm{~g}$ & $20,7 \mathrm{f}$ & 32,0 e \\
\hline BGH 5963 & $287,0 \mathrm{a}$ & $5,6 \mathrm{~g}$ & $19,7 \mathrm{~g}$ & $32,0 \mathrm{e}$ \\
\hline Vera Cruz 5004 & $280,0 \mathrm{a}$ & $5,6 \mathrm{~g}$ & $19,9 \mathrm{~g}$ & $31,0 \mathrm{e}$ \\
\hline Roxo Mineiro & $303,3 \mathrm{a}$ & $5,5 \mathrm{~g}$ & $18,1 \mathrm{~g}$ & $23,7 \mathrm{~g}$ \\
\hline BGH 5936 & $256,7 \mathrm{a}$ & $5,4 \mathrm{~g}$ & $21,2 \mathrm{f}$ & $31,0 \mathrm{e}$ \\
\hline Cajuru 2315 & $287,0 \mathrm{a}$ & $5,4 \mathrm{~g}$ & $18,8 \mathrm{~g}$ & 31,0 e \\
\hline Formosa 4713 & $280,0 \mathrm{a}$ & $5,4 \mathrm{~g}$ & $19,3 \mathrm{~g}$ & $29,0 \mathrm{f}$ \\
\hline Roxinho 5063 & 263,7 a & $5,3 \mathrm{~g}$ & $20,1 \mathrm{~g}$ & $27,7 \mathrm{f}$ \\
\hline BGH 4814 & $287,0 \mathrm{a}$ & $5,2 \mathrm{~g}$ & $18,0 \mathrm{~g}$ & $28,7 \mathrm{f}$ \\
\hline Amarante Embrapa & 310,3 a & $5,2 \mathrm{~g}$ & $16,7 \mathrm{~g}$ & $27,0 \mathrm{f}$ \\
\hline Santa Catarina Branco & $280,0 \mathrm{a}$ & $5,1 \mathrm{~g}$ & $18,3 \mathrm{~g}$ & $28,7 \mathrm{f}$ \\
\hline Andradas Manoel Lopes 1 & $280,0 \mathrm{a}$ & $5,1 \mathrm{~g}$ & $18,3 \mathrm{~g}$ & 32,3 e \\
\hline Roxo Araras & $240,3 \mathrm{a}$ & $5,1 \mathrm{~g}$ & $21,0 \mathrm{f}$ & 29,7 e \\
\hline Roxo de Araras 2 & 296,3 a & $5,0 \mathrm{~g}$ & $16,8 \mathrm{~g}$ & $28,7 \mathrm{f}$ \\
\hline Do Reino de Araras & 296,3 a & $4,9 \mathrm{~g}$ & $16,7 \mathrm{~g}$ & $28,0 \mathrm{f}$ \\
\hline Alho Preto & $303,3 \mathrm{a}$ & $4,5 \mathrm{~h}$ & $14,7 \mathrm{~h}$ & $26,3 f$ \\
\hline Mossoró & $287,0 \mathrm{a}$ & $4,5 \mathrm{~h}$ & $15,5 \mathrm{~h}$ & $41,7 \mathrm{~b}$ \\
\hline Assaí 3702 & 233,3 a & $4,4 \mathrm{~h}$ & $18,7 \mathrm{~g}$ & $24,0 \mathrm{~g}$ \\
\hline Canela de Ema & 263,7 a & $3,4 i$ & $12,8 \mathrm{i}$ & $20,7 \mathrm{~h}$ \\
\hline Gravataí & $280,0 \mathrm{a}$ & $3,1 \mathrm{i}$ & $11,0 \mathrm{i}$ & $26,7 \mathrm{f}$ \\
\hline C.V. (\%) & 8,6 & 7,8 & 8,2 & 5,1 \\
\hline $\mathrm{F}$ & $1,7^{*}$ & $42,9^{* *}$ & $46,1^{\star *}$ & $30,6^{* *}$ \\
\hline
\end{tabular}

${ }^{1}$ Médias seguidas pela mesma letra na coluna não diferem entre si (teste de Scott-Knott, $5 \%) ;{ }^{2}$ Baseado na média aritmética de 3 bulbos/parcela $\left(0,3 \mathrm{~m}^{2}\right)$, obtidos ao acaso; *Efeito de acessos $(\mathrm{P}<0,05)$; ** Efeito de acessos $(\mathrm{P}<0,01)$ o peso médio do bulbo no experimento em Tietê, no qual seis classes foram detectadas. $\mathrm{O}$ acesso Bulbilho Aéreo 2, com média de 55,8 g por bulbo, superou os demais. Na segunda classe destacou-se o acesso Piedade com média de 47,9 g por bulbo. O acesso Gigante de Curitibanos e Andradas Manoel Lopes 2, que produziram pesos médios de bulbo de 40,8 e 36,9 g, respectivamente, ocuparam a terceira classe (Tabela 1).

No experimento em Tietê foram obtidas seis classes para o diâmetro médio do bulbo, variável relacionada ao tamanho de bulbos selecionados. Os acessos Piedade, com 49,3 mm e Bulbilho Aéreo 2, com 48,9 mm, superaram os demais. Os acessos Gigante de Curitibanos, Andradas Manoel Lopes 2 e BGH 5952, com 44,6 mm, 42,3 mm e $39,8 \mathrm{~mm}$ respectivamente, situaram-se em segundo lugar.

As médias referentes ao número de bulbos por hectare, produtividade $\left(\mathrm{t} \mathrm{ha}^{-1}\right)$, peso médio do bulbo (g) e diâmetro médio do bulbo $(\mathrm{mm})$ referentes aos 50 acessos avaliados em Jundiaí, bem como o valor de $\mathrm{F}$ da análise de variância e a comparação pelo teste de Skott-Knott encontram-se na Tabela 2.

A análise de variância para o número de bulbos por hectare mostrou-se estatisticamente significativa para o experimento em Jundiaí. O teste de SkottKnott para esta variável detectou apenas uma classe, demonstrando não haver diferença entre o número de bulbos por hectare de cada acesso avaliado. A semelhança entre o número de bulbos por hectare era esperada em função da metodologia adotada no experimento e a uniformidade de brotação.

Para o experimento em Jundiaí foram detectadas 9 classes de produtividade. $\mathrm{O}$ acesso Bulbilho Aéreo 2 foi o mais produtivo com $13,1 \mathrm{t} \mathrm{ha}^{-1}$ superando os demais. Na classe seguinte estão os acessos Piedade e Gigante de Curitibanos, com magnitudes respectivas de 11,6 tha-1 e 11,4 $\mathrm{t} \mathrm{ha}^{-1}$ (Tabela 2). Estes acessos superam Lavinia 1632, que nos experimentos de Lisbão et al. (1991) e Lisbão et al. (1993), foi o mais produtiva em São Paulo, e que neste ensaio posicionou-se na terceira classe de produtividade, com 9,6 t ha ${ }^{-1}$, juntamente com outros acessos. 
O teste Skott-Knott detectou 9 classes para o peso médio do bulbo para os acessos cultivados em Jundiaí. O acesso Bulbilho Aéreo 2 superou os demais com média de 48,0 g por bulbo. Por sua vez, Piedade e Gigante de Curitibanos expressaram pesos médios de bulbo semelhantes na segunda classe, com valores de 40,5 g e 41,7 g, respectivamente (Tabela 2).

Para o diâmetro médio do bulbo, o maior valor em magnitude foi verificado para o acesso Piedade, com 47,3 mm, que não diferiu do acesso Gigante de Curitibanos, que expressou 45,3 $\mathrm{mm}$ de diâmetro. Estes acessos foram superiores em diâmetro aos acessos Mossoró e Andradas Manoel Lopes 2, que produziram diâmetro médio de bulbo de 41,7 $\mathrm{mm}$ e $40,7 \mathrm{~mm}$, respectivamente.

Houve semelhança de resultados entre os dois locais quando se comparam o peso médio do bulbo com a produtividade e com o diâmetro do bulbo. A exceção no experimento em Jundiaí (Tabela 2) foi o acesso Mossoró que apresentou baixa produtividade $\left(4,5 \mathrm{t} \mathrm{ha}^{-1}\right)$ e cabeça grande $(41,7 \mathrm{~mm})$.

Portanto, os melhores tamanhos de bulbo aliados à boa produtividade em Jundiaí foram alcançados pelos acessos Piedade e Gigante de Curitibanos. As produtividades foram maiores do que a produtividade normal para o alho em São Paulo, que é de 4 a 8 t/ha (LISBÃO et al., 1993; TAVARES et al., 1998). Apesar da produtividade do acesso
Bulbilho Aéreo 2 ter sido de $13,1 \mathrm{t} \mathrm{ha}^{-1}$, comercialmente o diâmetro médio do seu bulbo classifica o acesso em categoria inferior à dos acessos Piedade e Gigante de Curitibanos, em razão de variações de 36 a 45 mm do diâmetro serem classificadas como tamanho 4 , ao passo que variações de 46 a $55 \mathrm{~mm}$ classificam o genótipo como tamanho 5 (TRANI et al., 1997), o que é um fator importante no valor monetário para o alho.

Os acessos Piedade e Gigante de Curitibanos exibiram excelente desempenho para produtividade e qualidade do bulbo, nos dois locais avaliados. Em contraposição, os acessos Bulbilho Aéreo 2 e Andradas Manoel Lopes 2 apresentaram interação com o ambiente, o primeiro para o tamanho do bulbo e o segundo para a produtividade. Aos agricultores, recomenda-se o plantio experimental destes quatro acessos de alho, porque revelaram produtividade e qualidade superior em relação às cultivares tradicionalmente plantadas.

\section{LITERATURA CITADA}

CAMARGO FILHO, W.P. Área e produçãodos principais produtos da agropecária do estado de São Paulo: levantamento subjetiva estado de São Paulo. São Paulo, 2003. Disponívelem: <http://www.iea.sp.gov.br/out/ ibcoiea.php >. Acesso em: 27 ago. 2004.

CAMARGO FILHO, W.P.; TRANI, P.E.; SIQUEIRA, W.; BERNHARDT, L.W.; MAZZEI, A.R.; SILVA, C.G.; URYU, E.N.; CABRERA FILHO, J. Repensando a agricultura paulista - cadeias produtivas do alho, da cebola e condimentos. São Paulo, 86 p., 1996. (não publicado)
DALLAMARIA, G.C.M. A cultura do alho no Brasil. Batata Show, ano 3, n.6, p.32-33, 2003. FERREIRA, D.F. Programa Sisvar versão 4.3 (Build 45). DEX/UFLA, Lavras (MG). Disponível em : <http://www.dex.ufla.br/danielff/ dff02.htm>. Acesso em: 15 mar. 2005.

GOMES, F.P. Curso de estatística experimental. 4a. ed. São Paulo: Livraria Nobel, 1970, 430 p. INSTITUTO BRASILEIRO DE GEOGRAFIA E ESTATÍSTICA Produção Agrícola Municipal: Alho. Brasília, 2002. Disponível em: <http:// www.sidra.ibge.gov.br/bda/tabela/ protabl.asp?z=t\&o=1\&i=P>. Acesso em 27 ago. 2004.

LISBÃO, R.S.; FORNASIER, J.B.; GROPPO, G.A.; NEVES, J.P.S.; BARBOSA, L.A.V.; INADA, I.; RODRIGUES, A.; VERAGUAS, S.; FERNANDES, S.C.S. Avaliação de cultivares de alho. Campinas, Coordenadoria de Assistência Técnica Integral. Programa de Pesquisa e Adaptação de Tecnologias Regionais, n.22, mai. 1991. LISBÃO, R.S.; SIQUEIRA, W.J.; FORNASIER, J.B.; TRANI, P.E. Alho. In: FURLANI, A.M.C.; VIEGAS, G.P., Ed. O melhoramento de plantas no Instituto Agronômico. Campinas, Instituto Agronômico, 1993. p.222-253. 524 p.

SIQUEIRA, W.J.; TAVARES, M.; TRANI, P.E. Variedades de alho para o estado de São Paulo. Campinas, Instituto Agronômico, 1996. 26 p ( Boletim Técnico, 165)

TAVARES, M.; TRANI, P.E.; SIQUEIRA, W.J. Alho. In: Instruções agrícolas para as principais culturas econômicas, editado por Joel Irineu Fahl, Marcelo Bento Paes de Camargo, Maria Angélica Pizzinatto, Juarez Antonio Betti, Arlete Marchi Tavares de Melo, Isabela Clerici Demaria e Angela Maria Cangiani Furlani. 6. ed. rev. atual. Campinas, Instituto Agronômico, 1998. p.175-176. (Boletim IAC, 200)

TRANI, P.E.; TAVARES, M.; SIQUEIRA, W.J.; SANTOS, R.R.; BISÃO, L.G.; LISBÃO, R.S. Cultura do alho: recomendações para seu cultivo no estado de São Paulo. Campinas, Instituto Agronômico, 1997. 39 p (Boletim Técnico, 170) 